\title{
RANK ZERO AND RANK ONE SETS OF HARMONIC MAPS*
}

\author{
QING HAN ${ }^{\dagger}$ AND FANGHUA LIN ${ }^{\ddagger}$
}

Introduction. In this paper we study the sets of rank one and rank zero for harmonic maps between two Riemannian manifolds. The rank one sets are generalizations of nonsingular nodal sets of harmonic functions, while the rank zero sets are generalizations of critical sets, or more specific, critical nodal sets, of harmonic functions. Nodal sets and critical nodal sets are studied intensively for harmonic functions, or in general, solutions of general elliptic equations of the second order. In [22], Lin derived an optimal bound for the geometric measure of nodal sets for analytic solutions in terms their frequency. A less optimal bound was obtained for nonanalytic solutions in [19] by Hardt and Simon. In the same paper, Hardt and Simon proved that for classical solutions with relatively high order derivatives critical nodal sets are countable unions of subsets of correspondingly smooth $(n-2)$-dimensional submanifolds. Thus, they are countably $(n-2)$-rectifiable. See also [6]. This result was generalized to weak solutions in [15]. It is proved that for weak solutions, as long as they do not vanish to infinite order, the critical nodal sets are countable unions of subsets of $C^{1}$ $(n-2)$-dimensional submanifolds. Hence they are also countably $(n-2)$-rectifiable. Concerning the size of critical nodal sets, R. Hardt and the authors obtained a uniform estimate on the measure of critical nodal sets in terms of the frequency of solutions in [17]. See also a paper by M. and T. Hoffmann-Ostenhof, N. Nadirashvili and R. Hardt [21].

In this paper we will generalize those results to rank one and rank zero sets of harmonic maps. In [26] Sampson showed that when an open subset is mapped into an geodesic by a harmonic map then the whole domain is mapped into the geodesic. We will show in this paper that for a given harmonic map either the whole domain is mapped into a geodesic or the set of points where the differential has rank one is countably $(n-1)$-rectifiable. In the latter case we also obtain a measure estimate on this set. Rank zero sets were first studied by Gromov and Schoen [14] for minimizing harmonic maps when the image is in a singular space with the so-called nonpositive curvature property. They showed that such sets have codimensions at least two. Later on this result was generalized to arbitrary smooth harmonic maps between two smooth manifolds by S. Yamada [30]. In this paper we will show that rank zero sets of minimizing harmonic maps are countably $(n-2)$-rectifiable and have bounded $(n-2)$-dimensional Hausdorff measure. Our method is similar to that in [17].

The main results are as follows.

Theorem A. Suppose $(N, h)$ is a smooth compact Riemannian manifold and that $u: B_{1} \rightarrow N$ is a smooth harmonic map. Then the rank zero set $\mathcal{R}_{0}(u)$, the subset of $B_{1}$ where the differential du has rank zero, is countably $(n-2)$-rectifiable unless $u$ maps the whole $B_{1}$ to a point in $N$. Moreover there holds

$$
\mathcal{H}^{n-2}\left(\mathcal{R}_{0}(u) \cap B_{1 / 2}\right) \leq C
$$

\footnotetext{
* Received November 11, 1999; The authors are supported in part by NSF grants.

† Department of Mathematics, University of Notre Dame, Notre Dame, IN 46556, USA (qhan@ yansu.math.nd.edu).

$\ddagger$ Courant Institute, 251 Mercer Street, New York, NY 10012, USA (linf@math1.cims.nyu.edu).
} 
where the positive constant $C$ depends on the manifold $N$, the supnorm of the gradient of $u$ on $B_{1}$ and the quantity $\int_{B_{1}}\left|u-u_{1}\right|^{2}$ with $u_{1}$ as the average of $u$ over $B_{1}$.

THEOREM B. Suppose $(N, h)$ is a smooth compact Riemannian manifold and that $u: B_{1} \rightarrow N$ is a minimizing harmonic map. Then the rank zero set $\mathcal{R}_{0}(u)$ is countably $(n-2)$-rectifiable unless $u$ maps the whole $B_{1}$ to a point in $N$. Moreover there holds

$$
\mathcal{H}^{n-2}\left(\mathcal{R}_{0}(u) \cap B_{1 / 2}\right) \leq C
$$

where the positive constant $C$ depends on the manifold $N$, the total energy $\int_{B_{1}}|D u|^{2}$ of $u$ on $B_{1}$ and the quantity $\int_{B_{1}}\left|u-u_{1}\right|^{2}$ with $u_{1}$ as the average of $u$ over $B_{1}$. In particular if $\operatorname{sing}(u) \cap B_{2 / 3} \neq \phi$, then $C$ depends only on the manifold $N$ and the total energy $\int_{B_{1}}|D u|^{2}$ of $u$ on $B_{1}$. $C$.

In both theorems, the smaller the quantity $\int_{B_{1}}\left|u-u_{1}\right|^{2}$, the larger the constant

Concerning the rank one sets we have the following results. In the next two theorems, the quantity $J(u)$ is defined as (5.4) in Section 5. It describes quantitatively how harmonic maps differ from geodesic maps.

Theorem C. Suppose $(N, h)$ is a smooth compact Riemannian manifold and that $u: B_{1} \rightarrow N$ is a smooth harmonic map. Then the rank one set $\mathcal{R}_{1}(u)$, the subset of $B_{1}$ where the differential du has rank one, is countably $(n-1)$-rectifiable unless $u$ maps the whole $B_{1}$ to a point or a geodesic in $N$. Moreover there holds

$$
\mathcal{H}^{n-1}\left(\mathcal{R}_{1}(u) \cap B_{1 / 2}\right) \leq C
$$

where the positive constant $C$ depends on the manifold $N$, the supnorm of the gradient of $u$ on $B_{1}$ and the supnorm of $J(u)$.

THEOREM D. Suppose $(N, h)$ is a smooth compact Riemannian manifold and that $u: B_{1} \rightarrow N$ is a minimizing harmonic map. Then the rank one set $\mathcal{R}_{1}(u)$ is countably $(n-1)$-rectifiable unless $u$ maps the whole $B_{1}$ to a point or a geodesic in $N$. Moreover there holds

$$
\mathcal{H}^{n-1}\left(\mathcal{R}_{1}(u) \cap B_{1 / 2}\right) \leq C
$$

where the positive constant $C$ depends on the manifold $N$, the total energy $\int_{B_{1}}|D u|^{2}$ of $u$ on $B_{1}$ and the supnorm of $J(u)$ on $B_{1}$. In particular if $\operatorname{sing}(u) \cap B_{2 / 3} \neq \phi$, then $C$ depends only on the manifold $N$ and the total energy $\int_{B_{1}}|D u|^{2}$ of $u$ on $B_{1}$.

In both theorems, the smaller the supnorm of $J(u)$ on $B_{1}$, the larger the constant $C$.

The proofs of these theorems are similar. We use Theorems A and B for an illustration. They consist of three steps. The first step is the decomposition of the rank zero sets. We will show that, at almost all points in rank zero sets, the sets themselves are approximated by $(n-2)$-dimensional hyperplanes and harmonic maps are approximated by nonzero homogeneous harmonic polynomials of two variables by appropriate rotations. The second step is to show that when harmonic maps are approximated by nonzero homogeneous harmonic polynomials of two variables the rank zero sets have local measure estimates. This is based on a stability result of the splitting of zeroes for holomorphic functions, whose proof requires some knowledge of local rings. The last step is to iterate the local estimates obtained in step two. In order 
to achieve this we need to work in a class of smooth or minimizing harmonic maps which satisfies some nice compactness property. Because different compact classes are defined for smooth harmonic maps and minimizing harmonic maps, iteration processes are quite different. The discussion for minimizing harmonic maps is more complicated. In fact it requires the result established for smooth harmonic maps.

The paper consists of five sections. In the first section we discuss the geometric structure of rank zero sets. We will prove a stratification result which plays an important role in the subsequent sections. In the second section we will discuss the measure estimates of rank zero sets of smooth harmonic maps. Minimizing harmonic maps will be discussed in the third section. In the fourth section we will prove a stability lemma which is needed in sections two and three. In the last section we discuss the rank one sets. It is similar to that for rank zero sets. So the discussion is brief.

A major portion of the work in this paper was done while the first author was visiting Courant Institute. He would like to thank the Courant Institute for its hospitality. He would also like to thank the Slaon Foundation for the partial support.

1. Geometric Structure of Rank Zero Sets. First we assume that $B_{1}$ is the unit ball in $\mathbb{R}^{n}, n \geq 2$, endowed with a Riemannian metric $g$. Throughout this paper we always assume that $g$ is the standard Euclidean metric. The general case can be discussed in a similar fashion. We further assume that $(N, h)$ is a smooth compact Riemannian manifold of the dimension $m \geq 2$, which by Nash's isometric embedding theorem, we assume is isometrically embedded in some Euclidean space $\mathbb{R}^{k}$. We consider maps $u$ of $B_{1}$ into $N$; such maps will be thought of as maps $u=\left(u^{1}, \cdots, u^{k}\right)$ : $B_{1} \rightarrow \mathbb{R}^{k}$ with the additional property that $u\left(B_{1}\right) \subset N$. If $u$ is nonsmooth it is understood as $u(x) \in N$ for a.e. $x \in B_{1} . H^{1}\left(B_{1}, N\right)$ will denote the set of maps $u \in H^{1}\left(B_{1}, \mathbb{R}^{k}\right)$ such that $u\left(B_{1}\right) \subset N$. For any $u \in H^{1}\left(B_{1}, N\right)$ we define the Dirichlet energy of $u$ as

$$
E\left(u, B_{1}\right)=\int_{B_{1}}|D u|^{2} .
$$

The map $u \in H^{1}\left(B_{1}, N\right)$ is called a minimizing harmonic map if $E\left(u, B_{1}\right) \leq E\left(v, B_{1}\right)$ for any $v \in H^{1}\left(B_{1}, N\right)$ with $u=v$ on $\partial B_{1}$.

If $u \in H^{1}\left(B_{1}, N\right)$ is a minimizing harmonic map, then $u$ satisfies the following elliptic system in the weak sense

$$
\triangle u+A(u)(D u, D u)=0 \quad \text { in } B_{1}
$$

where $\triangle$ is the usual Laplacian operator in $B_{1}$ and $A(u)$ denotes the second fundamental form of $N$ at the point $u \in N$. In general the smooth solutions of this elliptic system is called smooth harmonic maps.

Given a smooth harmonic map $u: B_{1} \rightarrow N$, we define the rank zero set $\mathcal{R}_{0}(u)$ as

$$
\mathcal{R}_{0}(u)=\left\{p \in B_{1} ; D u(p)=0\right\} .
$$

For a minimizing harmonic map $u: B_{1} \rightarrow N$, we define the rank zero set $\mathcal{R}_{0}(u)$ as

$$
\mathcal{R}_{0}(u)=\{p \in \operatorname{reg}(u) ; D u(p)=0\},
$$

where $\operatorname{reg}(u)$ denotes the regular set of $u$, the subset of $B_{1}$ where $u$ is smooth. A well-known theorem of Schoen and Uhlenbeck [27] shows that

$$
\operatorname{dim}_{\mathcal{H}}\left(B_{1} \backslash \operatorname{reg}(u)\right) \leq n-3
$$


where $\operatorname{dim}_{\mathcal{H}}$ denotes the Hausdorff dimension.

The main result in this section is the following theorem.

THEOREM 1.1. Suppose $u \in H^{1}\left(B_{1}, N\right)$ is a smooth harmonic map or a minimizing harmonic map. Then the rank zero set $\mathcal{R}_{0}(u)$ is countably $(n-2)$-rectifiable unless $u$ maps the whole $B_{1}$ to a point in $N$.

Proof. Since this is a local result, we may assume that $u$ is smooth in $B_{1}$. Hence $u$ satisfies the elliptic system

$$
\triangle u+A(u)(D u, D u)=0 \quad \text { in } B_{1} .
$$

By the unique continuation property of the second-order elliptic systems, (see [2]), $u$ cannot vanish to infinite order unless $u$ is a constant. In the following we always assume that $u$ is not a constant map.

For each integer $d \geq 2$, define the rank zero set of the $d$-th level

$$
\mathcal{R}_{0}^{d}(u)=\left\{y \in B_{1} ; D^{i} u(y)=0, \text { for any } i=1, \cdots, d-1, \quad D^{d} u(y) \neq 0\right\} .
$$

Since $u$ does not vanish to infinite order, $\mathcal{R}_{0}^{d}(u)=\phi$ for sufficiently large $d$. Therefore

$$
\mathcal{R}_{0}(u)=\bigcup_{d \geq 2} \mathcal{R}_{0}^{d}(u)
$$

Take any point $y \in B_{1}(0) \cap \mathcal{R}_{0}^{d}(u)$. Consider the Taylor expansion of $u$ at $y$. By the definition $\mathcal{R}_{0}^{d}(u)$, the lowest nontrivial term in the expansion, except possibly the constant term, is given by a homogeneous polynomial $P=P_{y}: \mathbb{R}^{n} \rightarrow \mathbb{R}^{k}$ of degree $d$.

From the equation (1.3), $\triangle u$ vanishes at the point $y$ to the order at least $d-2$ since $u$ is of degree at least $d$. On the other hand, the right hand side vanishes to the order at least $(d-1)+(d-1)=2 d-2$, since $D u$ vanishes to the order at least $d-1$. Since $d-2 \leq 2 d-2$ under the condition $d \geq 2$, the $d$-th degree homogeneous terms of $u$ at $y$ are harmonic, i.e., $\triangle P=0$.

To summarize, for any $y \in \mathcal{R}_{0}^{d}(u)$, there exists a nontrivial homogeneous polynomial $P=P_{y}: \mathbb{R}^{n} \rightarrow \mathbb{R}^{k}$ of degree $d$ such that

$$
u(x+y)=u(y)+P(x)+o\left(|x|^{d}\right) \quad \text { as } x \rightarrow 0
$$

and

$$
\triangle P=0
$$

We should point out that some components of $P$ might be zero, but there is at least one nontrivial component. For convenience, we will call $P=P_{y}$ the leading polynomial of $u$ at $y$. With the introduction of leading polynomials, the rank zero set of the $d$-th level can be written alternatively as

$$
\mathcal{R}_{0}^{d}(u)=\left\{y \in B_{1} ; \operatorname{deg} P_{y}=d\right\} .
$$

In the following we will show that each $\mathcal{R}_{0}^{d}(u)$ is countably $(n-2)$-rectifiable.

Take any point $y \in \mathcal{R}_{0}^{d}(u)$ and assume the leading polynomial of $u$ at $y$ is given by the $d$-degree non-zero homogeneous polynomial $P=P_{y}$. Then $P$ satisfies $\triangle P=0$ in $\mathbb{R}^{n}$. For $P=\left(P^{1}, \cdots, P^{k}\right)$, if $P^{\alpha}$ is nontrivial for some $\alpha=1, \cdots, k$, we set

$$
\mathcal{R}^{d}\left(P^{\alpha}\right)=\left\{x \in B_{1} ; D^{i} P^{\alpha}(x)=0, \text { for any } i=0,1, \cdots, d-1, \quad D^{d} P^{\alpha}(x) \neq 0\right\} .
$$

Since $P^{\alpha}$ is a $d$-degree non-zero homogeneous polynomial, we always have $0 \in \mathcal{R}^{d}\left(P^{\alpha}\right)$. 
We claim that $\mathcal{R}^{d}\left(P^{\alpha}\right)$ is a linear subspace and

$$
P^{\alpha}(x)=P^{\alpha}(x+z) \text { for any } x \in \mathbb{R}^{n} \text { and } z \in \mathcal{R}^{d}\left(P^{\alpha}\right) .
$$

In fact for any $z \in \mathcal{R}^{d}\left(P^{\alpha}\right)$, we have

$$
D^{\nu} P^{\alpha}(z)=0 \text { for any }|\nu| \leq d-1 .
$$

Assume

$$
P^{\alpha}(x)=\sum_{|\alpha|=d} a_{\alpha} x^{\alpha}
$$

Then we have

$$
P^{\alpha}(x)=\sum_{|\alpha|=d} a_{\alpha}(x-z)^{\alpha}
$$

This implies (1.4). Now we may prove easily that $\mathcal{R}^{d}\left(P^{\alpha}\right)$ is a linear subspace.

Next, we show that $\operatorname{dim} \mathcal{R}^{d}\left(P^{\alpha}\right) \leq n-2$ for any $d \geq 2$. In fact the formula (1.4) implies $P^{\alpha}$ is a function of $n-\operatorname{dim} \mathcal{R}^{d}\left(\bar{P}^{\alpha}\right)$ variables. If $\operatorname{dim} \mathcal{R}^{d}\left(P^{\alpha}\right)=n-1$, then $P^{\alpha}$ would be a $d$-degree harmonic monomial of one variable. Hence $d=0$ or 1 , which is a contradiction.

We now define for $\alpha=1, \cdots k$, and $j=0,1,2, \cdots, n-2$,

$$
\mathcal{R}_{\alpha, j}^{d}(u)=\left\{y \in \mathcal{R}^{d}(u) ; \operatorname{dim} \mathcal{R}^{d}\left(P_{y}^{\alpha}\right)=j\right\} .
$$

We claim that $\mathcal{R}_{\alpha, j}^{d}(u)$ is on a countable union of $j$-dimensional $C^{1}$ manifolds for any $d \geq 2, \alpha=1, \cdots k$, and $j=0,1,2, \ldots, n-2$. In fact we will prove that for any $y \in \mathcal{R}_{\alpha, j}^{d}(u)$ there exists an $r=r(y)$ such that $\mathcal{R}_{\alpha, j}^{d}(u) \cap B_{r}(y)$ is contained in a $j$-dimensional $C^{1}$ manifold.

To show this we let $\ell_{y}^{\alpha}$ be the $j$-dimensional linear subspace $\mathcal{R}^{d}\left(P_{y}^{\alpha}\right)$ for any $y \in$ $\mathcal{R}_{\alpha, j}^{d}(u)$. We assume $y=0$ and $u(y)=0$ for simplicity. For any $\left\{y_{l}\right\} \subset \mathcal{R}_{\alpha, j}^{d}(u)$ with $y_{l} \rightarrow y$, we may assume by taking subsequence if necessary that $\xi_{l}=y_{l} /\left|y_{l}\right| \rightarrow \xi \in \mathbb{S}_{1}$. Since $P=P_{y}$ is the leading polynomial of $u$ at $y=0$, there holds for $i=0,1, \cdots, d-1$,

$$
D^{i}(u(x)-P(x))=o\left(|x|^{d-i}\right) \quad \text { as } x \rightarrow 0 .
$$

Evaluating at $y_{l}=\left|y_{l}\right| \xi_{l}$ and taking the limit $l \rightarrow \infty$, we conclude that $D^{i} P(\xi)=0$ for any $i=0,1, \cdots, d-1$. Since $P^{\alpha}$ is a nontrivial $d$-degree homogeneous polynomial, then $D^{d} P^{\alpha}(\xi) \neq 0$, and hence $\xi \in \ell_{y}^{\alpha}=\mathcal{S}^{d}\left(P_{y}^{\alpha}\right)$. Therefore we conclude

$$
\text { Angle }<\overline{y y_{l}}, \ell_{y}^{\alpha}>\rightarrow 0
$$

whenever $\left\{y_{l}\right\} \subset \mathcal{R}_{\alpha, j}^{d}(u)$ with $y_{l} \rightarrow y \in \mathcal{R}_{\alpha, j}^{d}(u)$. Furthermore let $P_{l}$ and $P$ be the leading polynomials of $u$ at $y_{l}$ and $y=0$, respectively. By smoothness of the map $u$ we have

$$
P_{l} \rightarrow P \text { uniformly in } C^{d}\left(B_{1}(0)\right) \text {. }
$$

This implies that

$$
\ell_{y_{l}}^{\alpha} \rightarrow \ell_{y}^{\alpha} \quad \text { as } l \rightarrow \infty
$$

as subspaces in $\mathbb{R}^{n}$. 
By (1.5) we obtain that for any $y \in \mathcal{R}_{\alpha, j}^{d}(u)$ and small $\varepsilon>0$ there exists an $r=r(y, \varepsilon)$ such that

$$
\mathcal{R}_{\alpha, j}^{d} \cap B_{r}(y) \subset B_{r}(y) \cap C_{\varepsilon}\left(\ell_{y}^{\alpha}\right)
$$

where

$$
C_{\varepsilon}\left(\ell_{y}^{\alpha}\right)=\left\{z \in \mathbb{R}^{n} ; \operatorname{dist}\left(z, \ell_{y}^{\alpha}\right) \leq \varepsilon|z|\right\} .
$$

Moreover by an argument similar to above to prove (1.5) we may prove that the constant $r$ in (1.6) can be chosen uniformly for any point $z \in \mathcal{R}_{\alpha, j}^{d}(u)$ in a neighborhood of $y$. In other words we obtain that for any $y \in \mathcal{R}_{\alpha, j}^{d}(u)$ and any small $\varepsilon>0$ there exists an $r=r(\varepsilon, y)$ such that

$$
\mathcal{R}_{\alpha, j}^{d}(u) \cap B_{r}(z) \subset B_{r}(z) \cap C_{\varepsilon}\left(\ell_{z}^{\alpha}\right) \quad \text { for any } z \in \mathcal{R}_{\alpha, j}^{d}(u) \cap B_{r}(y) .
$$

For $\varepsilon>0$ small enough this clearly implies that $\mathcal{R}_{\alpha, j}^{d}(u) \cap B_{r}(y)$ is contained in a $j$-dimensional Lipschitz manifold. By (1.5) this manifold is $C^{1}$.

We may define for $j=0,1, \cdots, n-2$,

$$
\mathcal{R}_{0, j}(u)=\bigcup_{d \geq 2} \bigcup_{\alpha=1}^{k} \mathcal{R}_{\alpha, j}^{d}(u) .
$$

Then we have

$$
\mathcal{R}_{0}(u)=\bigcup_{j=0}^{n-2} \mathcal{R}_{0, j}(u) .
$$

Moreover each $\mathcal{R}_{0, j}(u)$ is on a countable union of $j$-dimensional $C^{1}$ manifolds for $j=0,1, \cdots, n-2$. This finishes the proof of Theorem 1.1. We should emphasize that $\mathcal{R}_{0,0}(u), \mathcal{R}_{0,1}(u), \cdots, \mathcal{R}_{0, n-2}(u)$ are not necessarily mutually disjoint and that they may have overlaps.

Remark. With the notation in the proof, we set

$$
\begin{aligned}
& \mathcal{R}_{\star}(u)=\bigcup_{j=0}^{n-3} \mathcal{R}_{0, j}(u) \\
& \mathcal{R}^{\star}(u)=\mathcal{R}_{0, n-2}(u) .
\end{aligned}
$$

Then we have the decomposition

$$
\mathcal{R}_{0}(u)=\mathcal{R}^{\star}(u) \cup \mathcal{R}_{\star}(u)
$$

with the property that $\mathcal{R}_{\star}(u)$ is countably $(n-3)$-rectifiable, that $\mathcal{R}^{\star}(u)$ is on a countable union of $(n-2)$-dimensional $C^{1}$ manifolds and that for any $y \in \mathcal{R}^{\star}(u)$ some component $P_{y}^{\alpha}$ of the leading polynomial $P_{y}$ of $u$ at $y$ is a homogeneous harmonic polynomial of 2 variables. Such a decomposition will play an important role in the following sections.

2. Measure Estimates of Rank Zero Sets of Smooth Harmonic Maps. In this section we discuss the measure of rank zero sets of smooth harmonic maps.

Suppose $u \in H^{1}\left(B_{1}, N\right)$ is a nonconstant smooth harmonic map. Then $u$ satisfies the following elliptic system

$$
\triangle u+A(u)(D u, D u)=0 \quad \text { in } B_{1} .
$$


We first point out some obvious facts about smooth harmonic maps. For any positive constant $M$ we use $\mathcal{H}(M)$ to denote the smooth harmonic maps $u: B_{1} \rightarrow N$ such that

$$
\sup _{B_{1}}|D u| \leq M
$$

Lemma 2.1. The class $\mathcal{H}(M)$ is compact under the local $L^{2}$-metric.

Proof. The proof is straightforward. For any $u \in \mathcal{H}(M)$, by interior Schauder estimates we get for any positive integer $k$ and constant $r, \alpha \in(0,1)$

$$
\|u\|_{C^{k, \alpha}\left(B_{1-r}\right)} \leq C
$$

where $C$ is a positive constant depending only on the manifold $N$ and the constants $M, k, \alpha$ and $r$. Now consider a sequence $u_{i} \in \mathcal{H}(M)$. Then there exists a subsequence $u_{i^{\prime}}$ such that $u_{i^{\prime}}$ converges to some $u$ in $L^{2}$. It is easy to see by $(2.2)$ that in fact

$$
u_{i^{\prime}} \rightarrow u \text { in local } C^{k}\left(B_{1}\right)
$$

for any $k \geq 1$. Hence $u$ is smooth in $B_{1}$ and the image of $u$ lies on the manifold $N$. By taking $k=2$ we conclude that $u$ also satisfies the equation (2.1) and hence a smooth harmonic maps. It is obvious that $u$ belongs to the class $\mathcal{H}(M)$. This concludes the proof.

Remark. In fact we have the following result. For any two smooth harmonic maps $u$ and $v$ in $\mathcal{H}(M)$ if $\|u-v\|_{L^{2}\left(B_{1}\right)} \leq \eta$ for some positive $\eta>0$ then for any integer $k \geq 1$ and any $r \in(0,1)$ there holds

$$
\|u-v\|_{C^{k}\left(B_{1-r}\right)} \leq C(\eta)
$$

where $C(\eta)$ is a positive constant depending on $\eta$, as well as the manifold $N$ and the constants $M$ and $k$, with the property that $C(\eta) \rightarrow 0$ as $\eta \rightarrow 0$. This fact will be used in the following discussion.

For the next result we introduce the following standard notation. Define $u_{x, r}$ as the average of $u$ over $\partial B_{r}(x)$. For $x=0$ we simply write $u_{r}=u_{x, r}$.

Lemma 2.2. There exist positive constant $C$ and $r_{0}$ depending only on the manifold $N$ and the constant $M$ such that for any $x \in B_{1 / 2}$ and $r \in\left(0, r_{0}\right)$ there holds

$$
\frac{r \int_{B_{r}(x)}|D u|^{2}}{\int_{\partial B_{r}(x)}\left|u-u_{x, r}\right|^{2}} \leq C \frac{\int_{B_{1}}|D u|^{2}}{\int_{\partial B_{1}}\left|u-u_{1}\right|^{2}} .
$$

Lemma 2.2 is based on a variant of monotonicity formula, which claims that for some strictly positive function $c(x)$ the ratio

$$
\frac{r \int_{B_{r}(x)}|D u|^{2}}{\int_{\partial B_{r}(x)} c\left|u-u_{x, r}\right|^{2}}
$$

is a monotonically increasing function of $r \in(0,1-|x|)$. Such a monotonicity formula was proven for elliptic equations by Garofalo and Lin in [11]. The argument in [11] can be modified to yield the results in Lemma 2.2. The key observation is that under the assumption $|D u| \leq M$ in $B_{1}$ the elliptic system (2.1) can be viewed as a linear system with bounded coefficients for first order terms. Moreover the principal part of 
this system is decoupled. We will not provide the argument in detail. We refer the interesting readers to [11].

In view of Lemma 2.2 , we introduce $\mathcal{H}(M, F)$ for some positive constant $F$ as the collection of those harmonic maps $u \in \mathcal{H}(M)$ with the additional assumption

$$
\frac{r \int_{B_{r}(x)}|D u|^{2}}{\int_{\partial B_{r}(x)}\left|u-u_{x, r}\right|^{2}} \leq F
$$

for any $x \in B_{1 / 2}$ and $r \in(0,1 / 2)$.

Lemma 2.3. The class $\mathcal{H}(M, F)$ is compact under the local $L^{2}$-metric. Moreover the class $\mathcal{H}(M, F)$ is invariant under the translation and dilation. In other words, if $u \in \mathcal{H}(M, F)$, then for any $x \in B_{1 / 2}$ and $r \in(0,1 / 2)$ the map $u_{B_{r}(x)}$ also belong to $\mathcal{H}(M, F)$, where $u_{B_{r}(x)}(y)=u(x+r y)$ for $y \in B_{1}$.

This follows from Lemma 2.1 easily.

The main result of this section is the following theorem.

Theorem 2.4. For any $u \in \mathcal{H}(M, F)$, there holds

$$
\mathcal{H}^{n-2}\left(\mathcal{R}_{0}(u) \cap B_{1 / 2}\right) \leq C(N, M, F)
$$

where $C(N, M, F)$ is a positive constant depending only on the manifold $N$ and the constants $M$ and $F$.

Theorem A follows from Lemma 2.3 and Theorem 2.4 easily.

To prove Theorem 2.4 we first prove a lemma.

LEMma 2.5. For any $u \in \mathcal{H}(N, M, F)$, there exists a finite collection of balls $\left\{B_{r_{i}}\left(x_{i}\right)\right\}$ with $x_{i} \in \mathcal{R}_{0}(u)$ and $r_{i} \leq 1 / 4$ such that

$$
H^{n-2}\left(\mathcal{R}_{0}(u) \cap B_{1 / 2} \backslash \cup B_{r_{i}}\left(x_{i}\right)\right) \leq C,
$$

and

$$
\sum r_{i}^{n-2} \leq 1 / 2
$$

where $C$ is a positive constant depending only on the manifold $N$ and the constants $M$ and $F$, independent of $u$.

Proof. Take any $u \in \mathcal{H}(N, M, F)$. By Theorem 1.1 we have

$$
\mathcal{R}_{0}(u)=\mathcal{R}^{\star}(u) \cup \mathcal{R}_{\star}(u)
$$

where $\mathcal{R}_{\star}(u)$ has the Hausdorff dimension not exceeding $n-3, \mathcal{R}^{\star}(u)$ is on a countable union of $(n-2)$-dimensional $C^{1}$ manifolds and for any $y \in \mathcal{R}^{\star}(u)$ some component $P_{y}^{\alpha}$ of the leading polynomial $P_{y}$ of $u$ at $y$ is a homogeneous polynomial of 2 variables by an appropriate rotation. In particular

$$
H^{n-2}\left(\mathcal{R}_{\star}(u)\right)=0 .
$$

Then there exist at most countably many balls $B_{r_{i}}\left(x_{i}\right)$ with $r_{i} \leq 1 / 8$ and $x_{i} \in \mathcal{R}_{\star}(u)$ such that

$$
\mathcal{R}_{\star}(u) \subset \bigcup_{i} B_{r_{i}}\left(x_{i}\right)
$$


and

$$
\sum r_{i}^{n-2} \leq \frac{1}{2^{n-1}}
$$

We claim for any $y \in \mathcal{R}^{\star}(u) \cap B_{3 / 4}$, there exist positive constants $R=R(y, u)<$ $1 / 8, r=r(y, u)<R, \eta=\eta(y, u), c=c(y, u)$ and positive integer $K=K(y, u)$ such that if the function $v$ satisfies

$$
|u-v|_{C^{K}\left(B_{R}(y)\right)}^{*}<\eta
$$

then

$$
H^{n-2}\left\{\mathcal{R}_{0}(v) \cap B_{r}(y)\right\} \leq c r^{n-2} .
$$

Here we use $\|\cdot\|_{C^{M}\left(B_{R}\right)}^{*}$ to denote, $M$ as a positive integer, the $C^{M}$-norm weighted with the radius $R$, i.e., for $w \in C^{M}\left(B_{R}\right)$,

$$
\|w\|_{C^{M}\left(B_{R}\right)}^{*}=\sum_{i=0}^{M} R^{i} \sup _{x \in B_{R}}\left|D^{i} w(x)\right| .
$$

To prove (2.6) we take any $y \in \mathcal{R}^{\star}(u) \cap B_{3 / 4}$. By assuming $u(y)=0$, we have

$$
u(x+y)=P(x)+\psi(x)
$$

where $P=\left(P^{1}, \cdots, P^{k}\right)$ is a nonzero $d$-degree homogeneous harmonic polynomial with $d \geq 2$ and $\psi=\left(\psi^{1}, \cdots, \psi^{k}\right)$ is smooth in a neighborhood of the origin and satisfies

$$
D^{i} \psi(0)=0 \text { for } i=0,1, \cdots, d .
$$

For some $\alpha=1, \cdots, k, P^{\alpha}$ is a nontrivial homogeneous harmonic polynomial with $d \geq 2$ and by an appropriate rotation $P^{\alpha}$ is a polynomial of two variable. This follows from the remark at the end of section 1 . Hence we may apply Lemma 4.1 in the next section to $Q=P^{\alpha}$. Let $\varepsilon_{\star}$ and $r_{\star}$ be the constants given in Lemma 4.1 for $Q$. By (2.7) and the smoothness of $\psi$ at 0 we may take a positive constant $R=R(y, u)<1 / 8$ such that

$$
\left\|\frac{1}{R^{d}} \psi^{\alpha}\right\|_{C^{2 d^{2}\left(B_{R}\right)}}^{*}<\frac{1}{2} \varepsilon_{\star} .
$$

Choose $\eta$ small, depending on $R$ and $\varepsilon_{\star}$, such that (2.5), with $K=2 d^{2}$, implies

$$
\left\|\frac{1}{R^{d}}\left(u^{\alpha}-v^{\alpha}\right)\right\|_{C^{2 d^{2}}\left(B_{R}(y)\right)}^{*}<\frac{1}{2} \varepsilon_{\star} .
$$

Then there holds

$$
\left\|\frac{1}{R^{d}}\left(v^{\alpha}-Q(\cdot-y)\right)\right\|_{C^{2 d^{2}}\left(B_{R}(y)\right)}^{*}<\varepsilon_{\star} .
$$

By considering the transformation $x \mapsto y+R x$, we have

$$
\left\|\frac{1}{R^{d}} v^{\alpha}(y+R \cdot)-Q\right\|_{C^{2 d^{2}}\left(B_{1}\right)}<\varepsilon_{\star} .
$$

Note $Q$ is a homogeneous harmonic polynomial of two variables and of degree $d$. Hence we may apply Lemma 4.1 to $Q$. After transforming back to $B_{R}(y)$ we get for some $r \leq R r_{\star}$

$$
\mathcal{H}^{n-2}\left(\left|D v^{\alpha}\right|^{-1}\{0\} \cap B_{r}\right) \leq c(n)(d-1)^{2} r^{n-2} .
$$


Note that $\mathcal{R}_{0}(u) \cap B_{r}(y) \subset\left|D v^{\alpha}\right|^{-1}\{0\} \cap B_{r}$. Therefore we obtain (2.6).

It is obvious that the collection of $\left\{B_{r_{i}}\left(x_{i}\right)\right\}$ and $\left\{B_{r(y)}(y)\right\}, y \in \mathcal{R}^{\star}(u)$, covers $\mathcal{R}_{0}(u)$. By the compactness of $\mathcal{R}_{0}(u) \cup \operatorname{sing}(u)$, there exist $x_{i} \in \mathcal{R}_{\star}(u) \cup \operatorname{sing}(u)$, $i=1, \cdots, p=p(u)$, and $y_{j} \in \mathcal{R}^{\star}(u), j=1, \cdots, q=q(u)$, such that

$$
\mathcal{R}_{0}(u) \cap B_{3 / 4} \subset\left(\bigcup_{i=1}^{p} B_{r_{i}}\left(x_{i}\right)\right) \bigcup\left(\bigcup_{j=1}^{q} B_{s_{j}}\left(y_{j}\right)\right)
$$

with $r_{i} \leq 1 / 8, i=1, \cdots, p$, and $s_{j} \leq 1 / 8, j=1, \cdots, q$. Denote

$$
\mathcal{B}_{u}=\bigcup_{i=1}^{p} B_{r_{i}}\left(x_{i}\right), \quad \mathcal{G}_{u}=\bigcup_{j=1}^{q} B_{s_{j}}\left(y_{j}\right)
$$

Since $\mathcal{R}_{0}(u)$ is closed, there exists a positive constant $\rho=\rho(u)$ such that

$$
\left\{x \in B_{3 / 4} ; \operatorname{dist}(x, \mathcal{S}(u) \cup \sin g(u))<\rho\right\} \subset \mathcal{B}_{u} \cup \mathcal{G}_{u} .
$$

Consider any $v \in \mathcal{H}(N, M, F)$ with $\|u-v\|_{L^{2}\left(B_{1}\right)}<\delta$ for some constant $\delta>0$. By a priori estimates for elliptic system and a compactness argument there holds for any positive integer $\mathrm{K}$,

$$
|u-v|_{C^{K}\left(B_{3 / 4}\right)} \leq C(\delta)
$$

where $C(\delta) \rightarrow 0$ as $\delta \rightarrow 0$. See the remark after Lemma 2.1. Now we take

$$
K=K(u)=\max \left\{K\left(y_{1}, u\right), \cdots, K\left(y_{q}, u\right)\right\} .
$$

Obviously if $C(\delta)$ is small, then

$$
\mathcal{R}_{0}(v) \cap B_{1 / 2} \subset \mathcal{B}_{u} \cup \mathcal{G}_{u} .
$$

Moreover by taking $\delta=\delta(u)$ small further, we get that the condition (2.9) implies that for each $j=1, \cdots, q=q(u)$,

$$
|u-v|_{C^{K}\left(B_{R}\left(y_{j}\right)\right)}^{*}<\eta\left(y_{j}, u\right) .
$$

Hence by (2.6) we obtain for each $j=1, \cdots, q=q(u)$,

$$
\mathcal{H}^{n-2}\left(\mathcal{R}_{0}(v) \cap B_{s_{j}}\left(y_{j}\right)\right) \leq c s_{j}^{n-2} .
$$

Therefore there hold by (2.4), (2.9) and (2.10),

$$
\begin{gathered}
\mathcal{R}_{0}(v) \cap B_{1 / 2} \subset\left(\mathcal{R}_{0}(v) \cap \mathcal{B}_{u}\right) \cup\left(\mathcal{R}_{0}(v) \cap \mathcal{G}_{u}\right) \\
H^{n-2}\left(\mathcal{R}_{0}(v) \cap \mathcal{G}_{u}\right) \leq c \sum_{j=1}^{q} s_{j}^{n-2} \equiv C(u)
\end{gathered}
$$

and

$$
\mathcal{B}_{u}=\cup_{i=1}^{p} B_{r_{i}}\left(x_{i}\right), r_{i} \leq \frac{1}{8} \text { and } \sum_{i=1}^{k} r_{i}^{n-2} \leq \frac{1}{2^{n-1}} .
$$

If $\mathcal{R}_{0}(v) \cap B_{r_{i}}\left(x_{i}\right) \neq \phi$, we may take $\tilde{x}_{i} \in \mathcal{R}_{0}(v) \cap B_{r_{i}}\left(x_{i}\right)$. Obviously $B_{r_{i}}\left(x_{i}\right) \subset$ $B_{2 r_{i}}\left(\tilde{x}_{i}\right)$. Therefore, by renaming radii and centers, we get the following conclusion. For any $u \in \mathcal{H}(N, M, F)$, there exist positive constants $\delta=\delta(u)$ and $C=C(u)$ such 
that for any $v \in \mathcal{H}_{1}(N, M, F)$ with $\|u-v\|_{L^{2}\left(B_{1}\right)}<\delta$ there exist a finite collection of balls $\left\{B_{r_{i}}\left(x_{i}\right)\right\}$ with $x_{i} \in \mathcal{R}_{0}(v), r_{i} \leq 1 / 4$, and $\sum r_{i}^{n-2} \leq 1 / 2$ such that

$$
H^{n-2}\left(\mathcal{R}_{0}(v) \cap B_{1 / 2} \backslash \cup B_{r_{i}}\left(x_{i}\right)\right) \leq C(u) .
$$

By Lemma $2.3, \mathcal{H}(N, M, F)$ is compact under the $L^{2}$-metric. Hence there exist $u_{1}, \cdots, u_{L} \in \mathcal{H}_{1}(N, M, F)$ and $\delta_{1}=\delta\left(u_{1}\right), \cdots, \delta_{L}=\delta\left(u_{L}\right)$ such that for any $v \in$ $\mathcal{H}(N, M, F)$ there exists an $l, 1 \leq l \leq L$, with the property

$$
\left\|v-u_{l}\right\|_{L^{2}\left(B_{1}\right)}<\delta_{l} \text {. }
$$

Denote

$$
C=\max \left\{C\left(u_{1}\right), \cdots, C\left(u_{L}\right)\right\} .
$$

Such a $C$ depends only on the manifold $N$ and the constant $E_{0}$. This finishes the proof.

Now we prove Theorem 2.4.

Proof of Theorem 2.4. We use the standard iteration process. To begin with, define

$$
\phi_{0}=\left\{B_{1 / 2}(0)\right\} .
$$

We claim that we may find $\phi_{1}, \phi_{2}, \cdots$, each of which consists of a collection of balls, such that for any $\ell \geq 1$

$$
\begin{gathered}
\operatorname{rad}(B) \leq \frac{1}{2} \cdot\left(\frac{1}{2}\right)^{\ell} \quad \text { for any } B \in \phi_{\ell} \\
\sum_{B \in \phi_{\ell}}[\operatorname{rad}(B)]^{n-2} \leq\left(\frac{1}{2}\right)^{\ell}
\end{gathered}
$$

and

$$
H^{n-2}\left(\mathcal{R}_{0}(u) \cap \bigcup_{B \in \phi_{\ell-1}} B \sim \bigcup_{B \in \phi_{\ell}} B\right) \leq C\left(\frac{1}{2}\right)^{\ell-1}
$$

where $C$ is the positive constant given in Lemma 2.5. Observe that

$$
\begin{aligned}
\mathcal{R}_{0}(u) \cap B_{1 / 2}(0) \subset & \bigcup_{\ell=1}^{\infty}\left(\mathcal{R}_{0}(u) \cap\left(\bigcup_{B \in \phi_{\ell-1}} B \sim \bigcup_{B \in \phi_{\ell}} B\right)\right) \\
& \cup \bigcap_{\ell=0}^{\infty}\left(\mathcal{R}_{0}(u) \cap \bigcup_{j=\ell}^{\infty} \bigcup_{B \in \phi_{j}} B\right) .
\end{aligned}
$$

Hence we have

$$
H^{n-2}\left(\mathcal{R}_{0}(u) \cap B_{1 / 2}(0)\right) \leq C\left\{\sum_{\ell \geq 1}\left(\frac{1}{2}\right)^{\ell-1}+\inf _{\ell \geq 1} \sum_{j=\ell}^{\infty}\left(\frac{1}{2}\right)^{j}\right\} \leq 2 C .
$$

To prove the claim we construct $\left\{\phi_{\ell}\right\}$ by induction. Note $\phi_{0}=\left\{B_{1 / 2}\right\}$. Suppose $\phi_{0}, \phi_{1}, \ldots, \phi_{\ell-1}$ are already defined for some $\ell \geq 1$. To construct $\phi_{\ell}$ we take $B=$ $B_{r}(y) \in \phi_{\ell-1}$, with $r \leq 1 / 2$. Consider the transformation $x \mapsto y+2 r x$ and the map $u_{B}(x)=u(y+2 r x)$. By Lemma $2.4 u_{B} \in \mathcal{H}(N, M, F)$. Hence we may apply Lemma 
2.3 to $u_{B}$ to obtain a collection of balls $\left\{B_{s_{i}}\left(z_{i}\right)\right\}$, with $s_{i} \leq 1 / 4$ and $z_{i} \in \mathcal{R}_{0}\left(u_{B}\right)$ such that

$$
H^{n-2}\left(\mathcal{R}_{0}\left(u_{B}\right) \cap B_{1 / 2} \backslash \cup B_{s_{i}}\left(z_{i}\right)\right) \leq C
$$

and

$$
\sum s_{i}^{n-2} \leq \frac{1}{2}
$$

Now transform $B_{1 / 2}(0)$ back to $B_{r}(y)$ by $x \mapsto(x-y) / 2 r$. We obtain that for $B=$ $B_{r}(y) \in \phi_{\ell-1}$, there exist finitely many balls $\left\{B_{r_{i}}\left(x_{i}\right)\right\}$ in $B_{2 r}(y)$, with $r_{i} \leq 1 / 2$, such that

$$
H^{n-2}\left(\mathcal{R}_{0}(u) \cap B_{r}(y) \backslash \bigcup_{i} B_{r_{i}}\left(x_{i}\right)\right) \leq C r^{n-2},
$$

and

$$
\sum_{i} r_{i}^{n-2} \leq \frac{1}{2} r^{n-2}
$$

Then we set

$$
\phi_{\ell}^{B}=\bigcup_{i}\left\{B_{i}\left(x_{i}\right)\right\}
$$

and

$$
\phi_{\ell}=\bigcup_{B \in \phi_{\ell-1}} \phi_{\ell}^{B}
$$

Hence we obtain

$$
H^{n-2}\left(\mathcal{R}_{0}(u) \cap \bigcup_{B \in \phi_{\ell-1}} B \sim \bigcup_{B \in \phi_{\ell}} B\right) \leq C\left(\sum_{B_{r_{i}}\left(x_{i}\right) \in \phi_{\ell-1}} r_{i}^{n-2}\right)
$$

and by induction

$$
r_{i} \leq \frac{1}{2} \cdot\left(\frac{1}{2}\right)^{\ell}, \quad \sum_{B_{r_{i}}\left(x_{i}\right) \in \phi_{\ell}} r_{i}^{n-2} \leq\left(\frac{1}{2}\right)^{\ell}
$$

for each $\ell \geq 1$. This concludes the proof.

3. Geometric Measure of Rank Zero Sets of Minimizing Harmonic Maps. In this section we will discuss the measure estimates of rank zero sets of minimizing harmonic maps.

Suppose $u \in H^{1}\left(B_{1}, N\right)$ is a nonconstant minimizing harmonic map. We define

$$
\begin{aligned}
\operatorname{reg}(u) & =\left\{y \in B_{1} ; u \text { is smooth at } y\right\}, \\
\operatorname{sing}(u) & =B_{1} \backslash \operatorname{reg}(u)
\end{aligned}
$$

and

$$
\mathcal{R}_{0}(u)=\{y \in \operatorname{reg}(u) ; D u(y)=0\} .
$$

Schoen and Uhlenbeck [27] showed that $\operatorname{dim}_{\mathcal{H}}(\operatorname{sing}(u)) \leq n-3$. Theorem 1.1 implies that $\operatorname{dim}_{\mathcal{H}}\left(\mathcal{R}_{0}(u)\right) \leq n-2$.

The discussion in this section is more complicated than that in the previous section, partly because the set $\mathcal{R}_{0}(u)$ is only relatively closed in the open set $\operatorname{reg}(u)$. 
Another reason is the difficulty to construct a compact class of minimizing harmonic maps which is invariant by dilations.

For any positive constants $E$ and $m$, we use $\mathcal{H}_{m}(E, m)$ to denote the collection of minimizing harmonic maps $u: B_{1} \rightarrow N$ such that

$$
\int_{B_{1}}|D u|^{2} \leq E
$$

and

$$
|D u|_{L^{\infty}\left(B_{2 / 3}\right)} \geq m \text {. }
$$

First we prove

Lemma 3.1. The class $\mathcal{H}_{m}(E, m)$ is compact under the local $H^{1}$-metric.

Proof. The proof is straightforward as in the proof of Lemma 2.1. Suppose $u_{i} \in$ $\mathcal{H}_{m}(E, m)$ for any $i=1,2, \cdots$. Then it is obvious that there exists a subsequence $u_{i^{\prime}}$ such that $u_{i^{\prime}}$ converges $H^{1}$ weakly to some $u \in H^{1}\left(B_{1}, N\right)$, which is a minimizing harmonic map. Next we prove that

$$
|D u|_{L^{\infty}\left(B_{2 / 3}\right)} \geq m \text {. }
$$

It is obvious if $\operatorname{sing}(u) \cap B_{2 / 3} \neq \phi$. If $\operatorname{sing}(u) \cap B_{2 / 3}=\phi$, then by the small energy regularity theorem and the strong local $H^{1}$ convergence, we conclude that $\operatorname{sing}\left(u_{i^{\prime}}\right) \cap$ $B_{2 / 3}=\phi$ for all large $i^{\prime}$. Moreover there exists a positive constant $M>0$ such that

$$
\sup _{B_{2 / 3}}\left|D u_{i^{\prime}}\right| \leq M, \quad \text { for any } i^{\prime} .
$$

Hence the desired inequality follows from the uniform convergence of the gradients in $B_{2 / 3}$. See the remark after Lemma 3.1 .

The main result in this section is the following.

Theorem 3.2. For any $u \in \mathcal{H}_{m}(E, m)$, there holds

$$
\mathcal{H}^{n-2}\left(\mathcal{R}_{0}(u) \cap B_{1 / 2}\right) \leq C(N, E, m)
$$

where $C(N, E, m)$ is a positive constant depending only on the manifold $N$ and the constants $E$ and $m$.

We first prove a lemma.

Lemma 3.3. For any $u \in \mathcal{H}_{m}(E, m)$, there exists a finite collection of balls $\left\{B_{r_{i}}\left(x_{i}\right)\right\}$ with $x_{i} \in \mathcal{R}_{0}(u)$ and $r_{i} \in\left(r_{0}, 1 / 4\right)$ such that

$$
H^{n-2}\left(\mathcal{R}_{0}(u) \cap B_{1 / 2} \backslash \cup B_{r_{i}}\left(x_{i}\right)\right) \leq C
$$

and

$$
\sum r_{i}^{n-2} \leq 1 / 2
$$

where $C$ and $r_{0}$ are positive constants depending only on the manifold $N$ and the constants $E$ and $m$, independent of $u$.

Proof. The proof is similar to that of Lemma 2.6. So we only give details on the difference. Take any $u \in \mathcal{H}_{1}\left(E_{0}, N\right)$. By Theorem 1.1 we have

$$
\mathcal{R}_{0}(u)=\mathcal{R}^{\star}(u) \cup \mathcal{R}_{\star}(u)
$$


where $\mathcal{R}_{\star}(u)$ has the Hausdorff dimension not exceeding $n-3, \mathcal{R}^{\star}(u)$ is on a countable union of $(n-2)$-dimensional $C^{1}$ manifolds and for any $y \in \mathcal{R}^{\star}(u)$ some component $P_{y}^{\alpha}$ of the leading polynomial $P_{y}$ of $u$ at $y$ is a homogeneous polynomial of 2 variables by an appropriate rotation. In particular

$$
H^{n-2}\left(\mathcal{R}_{\star}(u) \cap \operatorname{sing}(u)\right)=0 .
$$

Then there exist at most countably many balls $B_{r_{i}}\left(x_{i}\right)$ with $r_{i} \leq 1 / 8$ and $x_{i} \in$ $\mathcal{R}_{\star}(u) \cap \operatorname{sing}(u)$ such that

$$
\mathcal{R}_{\star}(u) \cup \operatorname{sing}(u) \subset \bigcup_{i} B_{r_{i}}\left(x_{i}\right)
$$

and

$$
\sum r_{i}^{n-2} \leq \frac{1}{2^{n-1}}
$$

As in the proof of Lemma 2.6 we may prove that for any $y \in \mathcal{R}^{\star}(u) \cap B_{3 / 4}$, there exist positive constants $R=R(y, u)<1 / 8, r=r(y, u)<R, \eta=\eta(y, u), c=c(y, u)$ and positive integer $K=K(y, u)$ such that if the function $v$ satisfies

$$
|u-v|_{C^{K}\left(B_{R}(y)\right)}^{*}<\eta
$$

then

$$
H^{n-2}\left\{\mathcal{R}_{0}(v) \cap B_{r}(y)\right\} \leq c r^{n-2} .
$$

It is obvious that the collection of $\left\{B_{r_{i}}\left(x_{i}\right)\right\}$ and $\left\{B_{r(y)}(y)\right\}, y \in \mathcal{R}^{\star}(u)$, covers $\mathcal{R}_{0}(u) \cup \operatorname{sing}(u)$. By the compactness of $\mathcal{R}_{0}(u) \cup \operatorname{sing}(u)$, there exist $x_{i} \in \mathcal{R}_{\star}(u) \cup$ $\operatorname{sing}(u), i=1, \cdots, p=p(u)$, and $y_{j} \in \mathcal{R}^{\star}(u), j=1, \cdots, q=q(u)$, such that

$$
\left(\mathcal{R}_{0}(u) \cup \operatorname{sing}(u)\right) \cap B_{3 / 4} \subset\left(\bigcup_{i=1}^{p} B_{r_{i}}\left(x_{i}\right)\right) \bigcup\left(\bigcup_{j=1}^{q} B_{s_{j}}\left(y_{j}\right)\right)
$$

with $r_{i} \leq 1 / 8, i=1, \cdots, p$, and $s_{j} \leq 1 / 8, j=1, \cdots, q$. Moreover there holds

$$
\operatorname{sing}(u) \cap\left(\bigcup_{j=1}^{q} B_{s_{j}}\left(y_{j}\right)\right)=\phi .
$$

Denote

$$
\mathcal{B}_{u}=\bigcup_{i=1}^{p} B_{r_{i}}\left(x_{i}\right), \quad \mathcal{G}_{u}=\bigcup_{j=1}^{q} B_{s_{j}}\left(y_{j}\right) .
$$

Since $\mathcal{R}_{0}(u) \cup \operatorname{sing}(u)$ is closed, there exists a positive constant $\rho=\rho(u)$ such that

$$
\left\{x \in B_{3 / 4} ; \operatorname{dist}\left(x, \mathcal{R}_{0}(u) \cup \operatorname{sing}(u)\right)<\rho\right\} \subset \mathcal{B}_{u} \cup \mathcal{G}_{u}
$$

Define

$$
r(u)=\min \left\{2 r_{1}, \cdots, 2 r_{p}\right\} .
$$

Consider any $v \in \mathcal{H}_{1}\left(E_{0}, N\right)$ with $\|u-v\|_{H^{1}\left(B_{7 / 8}\right)}<\delta$ for some constant $\delta>0$. By taking $\delta$ small we get

$$
\left(B_{3 / 4} \backslash \mathcal{B}_{u}\right) \cup \mathcal{G}_{u} \subset \operatorname{reg}(v)
$$


and in particular

$$
\operatorname{sing}(v) \cap \mathcal{G}_{u}=\phi
$$

Moreover by a priori estimates for elliptic system and a compactness argument there holds for any positive integer $\mathrm{K}$,

$$
|u-v|_{C^{K}\left(\left(B_{3 / 4} \backslash \mathcal{B}_{u}\right) \cup \mathcal{G}_{u}\right)} \leq C(\delta)
$$

where $C(\delta) \rightarrow 0$ as $\delta \rightarrow 0$. Now we take

$$
K=K(u)=\max \left\{K\left(y_{1}, u\right), \cdots, K\left(y_{q}, u\right)\right\} .
$$

Obviously if $C(\delta)$ is small, then

$$
\mathcal{R}_{0}(v) \cap B_{1 / 2} \subset \mathcal{B}_{u} \cup \mathcal{G}_{u}
$$

Moreover by taking $\delta=\delta(u)$ small further, we get that the condition (3.7) implies that for each $j=1, \cdots, q=q(u)$,

$$
|u-v|_{C^{K}\left(B_{R}\left(y_{j}\right)\right)}^{*}<\eta\left(y_{j}, u\right) .
$$

Hence by (3.4) we obtain for each $j=1, \cdots, q=q(u)$,

$$
\mathcal{H}^{n-2}\left(\mathcal{R}_{0}(v) \cap B_{s_{j}}\left(y_{j}\right)\right) \leq c s_{j}^{n-2} .
$$

Therefore there hold by (3.2), (3.7) and (3.8),

$$
\begin{gathered}
\mathcal{R}_{0}(v) \cap B_{1 / 2} \subset\left(\mathcal{R}_{0}(v) \cap \mathcal{B}_{u}\right) \cup\left(\mathcal{R}_{0}(v) \cap \mathcal{G}_{u}\right) \\
H^{n-2}\left(\mathcal{R}_{0}(v) \cap \mathcal{G}_{u}\right) \leq c \sum_{j=1}^{q} s_{j}^{n-2} \equiv C(u)
\end{gathered}
$$

and

$$
\mathcal{B}_{u}=\cup_{i=1}^{p} B_{r_{i}}\left(x_{i}\right), r_{i} \leq \frac{1}{8} \text { and } \sum_{i=1}^{k} r_{i}^{n-2} \leq \frac{1}{2^{n-1}} .
$$

If $\mathcal{R}_{0}(v) \cap B_{r_{i}}\left(x_{i}\right) \neq \phi$, we may take $\tilde{x}_{i} \in \mathcal{R}_{0}(v) \cap B_{r_{i}}\left(x_{i}\right)$. Obviously $B_{r_{i}}\left(x_{i}\right) \subset$ $B_{2 r_{i}}\left(\tilde{x}_{i}\right)$. Therefore, by renaming radii and centers, we get the following conclusion. For any $u \in \mathcal{H}_{m}(E, m)$, there exist positive constants $\delta=\delta(u)$ and $C=C(u)$ such that for any $v \in \mathcal{H}_{m}(E, m)$ with $\|u-v\|_{H^{1}\left(B_{7 / 8}\right)}<\delta$ there exist a finite collection of balls $\left\{B_{r_{i}}\left(x_{i}\right)\right\}$ with $x_{i} \in \mathcal{R}_{0}(v), r_{i} \leq 1 / 4$, and $\sum r_{i}^{n-2} \leq 1 / 2$ such that

$$
H^{n-2}\left(\mathcal{R}_{0}(v) \cap B_{1 / 2} \backslash \cup B_{r_{i}}\left(x_{i}\right)\right) \leq C(u) .
$$

By Lemma 3.1, $\mathcal{H}_{m}(E, m)$ is compact under the local $H^{1}$-metric. Hence there exist $u_{1}, \cdots, u_{L} \in \mathcal{H}_{m}(E, m)$ and $\delta_{1}=\delta\left(u_{1}\right), \cdots, \delta_{L}=\delta\left(u_{L}\right)$ such that for any $v \in$ $\mathcal{H}_{m}(E, m)$ there exists an $l, 1 \leq l \leq L$, with the property

$$
\left\|v-u_{l}\right\|_{H^{1}\left(B_{7 / 8}\right)}<\delta_{l} .
$$

Denote

$$
\begin{gathered}
C=\max \left\{C\left(u_{1}\right), \cdots, C\left(u_{L}\right)\right\} \\
r_{0}=\max \left\{r\left(u_{1}\right), \cdots, r\left(u_{L}\right)\right\} .
\end{gathered}
$$


Such $C$ and $r_{0}$ depends only on the manifold $N$ and the constants $E$ and $m$. This finishes the proof.

Now we prove Theorem 3.2. As for Theorem 2.5 we use the standard iteration process. However the class $\mathcal{H}_{m}(E, m)$ is not invariant under the dilation. This makes the iteration process complicated.

Proof of Theorem 3.2. Take a map $u \in \mathcal{H}_{m}(E, m)$. To begin with, define

$$
\phi_{0}=\left\{B_{1 / 2}(0)\right\} \text {. }
$$

By Lemma 3.3 we find $\phi_{1}$, which consists of a collection of balls, such that

$$
\begin{gathered}
\operatorname{rad}(B) \leq \frac{1}{2} \cdot \frac{1}{2} \quad \text { for any } B \in \phi_{1} \\
\sum_{B \in \phi_{1}}[\operatorname{rad}(B)]^{n-2} \leq \frac{1}{2}
\end{gathered}
$$

and

$$
H^{n-2}\left(\mathcal{R}_{0}(u) \cap \bigcup_{B \in \phi_{0}} B \sim \bigcup_{B \in \phi_{1}} B\right) \leq C,
$$

where $C$ is the positive constant given in Lemma 3.3.

To illustrate the idea we only carry out the next step. We take any $B=B_{r}(y) \in$ $\phi_{1}$, with $y \in B_{1 / 2}$ and $r \leq 1 / 4$. Consider the transformation $x \mapsto y+2 r x$ and the map

$$
u_{B}(x)=u(y+2 r x) .
$$

Monotonicity formula implies

$$
\int_{B_{1}}\left|D u_{B}\right|^{2}=\frac{1}{(2 r)^{n-2}} \int_{B_{2 r}(y)}|D u|^{2} \leq 2^{n-2} \int_{B_{1 / 2}(y)}|D u|^{2} \leq 2^{n-2} E .
$$

We first consider the case that $\left|D u_{B}\right|_{L^{\infty}\left(B_{2 / 3}\right)} \leq m$. In this case $u_{B}$ is a smooth harmonic map in $B_{2 / 3}$. By Lemma 3.3 the radius of the ball $B$ satisfies $\operatorname{rad}(B)=r \geq$ $r_{0}$ for some positive number $r_{0}$ depending only on the manifold $N$ and the constants $E$ and $m$. Hence there exists a positive constant $\gamma$, again depending only on the manifold $N$ and the constants $E$ and $m$, such that

$$
\int_{B_{2 r}(y)}\left|u-u_{y, 2 r}\right|^{2} \geq \gamma
$$

where $u_{y, 2 r}$ is the average of $u$ on $B_{2 r}$. This follows easily from the compactness of the class $\mathcal{H}_{m}(E, m)$ and the continuity of the map $(y, r) \mapsto \int_{B_{2 r}(y)}\left|u-u_{y, 2 r}\right|^{2}$ for $B_{2 r}(y) \subset B_{1}$. Therefore there holds

$$
\frac{\int_{B_{1}}\left|D u_{B}\right|^{2}}{\int_{B_{1}}\left|u_{B}-\left(u_{B}\right)_{1}\right|^{2}} \leq F_{0}
$$

for some $F_{0}$ depending only on the manifold $N$ and the constants $E$ and $m$. Now we map apply Theorem $\mathrm{A}$ to $u_{B}$ to conclude that

$$
H^{n-2}\left(\mathcal{R}_{0}\left(u_{B}\right) \cap B_{1 / 2}\right) \leq C_{1}
$$


for some positive constant $C_{1}$ depending only on the manifold $N$ and the constants $E$ and $m$. Now transform $B_{1 / 2}(0)$ back to $B_{r}(y)$ by $x \mapsto(x-y) / 2 r$. We obtain that for $B=B_{r}(y) \in \phi_{1}$, if $u_{B}(x)=u(y+2 r x)$ satisfies

$$
\left|D u_{B}\right|_{L^{\infty}\left(B_{2 / 3}\right)} \leq m
$$

there holds

$$
H^{n-2}\left(\mathcal{R}_{0}(u) \cap B_{r}(y)\right) \leq C_{1} r^{n-2} .
$$

By (3.9) and (3.12) we conclude that

$$
\sum_{B \in \phi_{1} \text { satisfies }} H^{n-2}\left(\mathcal{R}_{0}(u) \cap \bigcup_{B \in \phi_{0}} B \sim \bigcup_{B \in \phi_{1}} B\right) \leq \frac{1}{2} C_{1} .
$$

Hence we may delete those balls satisfying (3.11) from the collection of $\phi_{1}$ and add the measure estimates (3.13) back to (3.10).

Therefore we obtain the following result. We still use the same notation. Recall

$$
\phi_{0}=\left\{B_{1 / 2}(0)\right\} .
$$

Then we can find $\phi_{1}$, which consists of a collection of balls, such that

$$
\begin{gathered}
\operatorname{rad}(B) \leq \frac{1}{2} \cdot \frac{1}{2} \text { for any } B \in \phi_{1} \\
\sum_{B \in \phi_{1}}[\operatorname{rad}(B)]^{n-2} \leq \frac{1}{2}
\end{gathered}
$$

and

$$
H^{n-2}\left(\mathcal{R}_{0}(u) \cap \bigcup_{B \in \phi_{0}} B \sim \bigcup_{B \in \phi_{1}} B\right) \leq C+\frac{1}{2} C_{1},
$$

where $C$ and $C_{1}$ are both positive constants depending only on the manifold $N$ and the constant $E$ and $m$. Moreover for any ball $B=B_{r}(y)$ the map $u_{B}(x)=u(y+2 r x)$ satisfies

$$
\left|D u_{B}\right|_{L^{\infty}\left(B_{2 / 3}\right)} \geq m \text {. }
$$

Now we may proceed inductively. We then obtain $\phi_{1}, \phi_{2}, \cdots$, each of which consists of a collection of balls, such that for any $\ell \geq 1$

$$
\begin{gathered}
\operatorname{rad}(B) \leq \frac{1}{2} \cdot\left(\frac{1}{2}\right)^{\ell} \quad \text { for any } B \in \phi_{\ell} \\
\sum_{B \in \phi_{\ell}}[\operatorname{rad}(B)]^{n-2} \leq\left(\frac{1}{2}\right)^{\ell}
\end{gathered}
$$

and

$$
H^{n-2}\left(\mathcal{R}_{0}(u) \cap \bigcup_{B \in \phi_{\ell-1}} B \sim \bigcup_{B \in \phi_{\ell}} B\right) \leq\left(\frac{1}{2}\right)^{\ell-1} C+\left(\frac{1}{2}\right)^{\ell} C_{1}
$$

where $C$ and $C_{1}$ are positive constants depending only on the manifold $N$ and the constants $E$ and $m$. 
The rest of the proof is the same as that in Theorem 2.5. This concludes the proof.

Now we prove Theorem B.

Proof of Theorem B. Suppose that $u \in H^{1}\left(B_{1}, N\right)$ is a minimizing harmonic map with $\int_{B_{1}}|D u|^{2} \leq E$. If $|D u|_{L^{\infty}\left(B_{1}\right)} \geq 1$, then we may apply Theorem 3.2 to conclude that

$$
\mathcal{H}^{n-2}\left(\mathcal{R}_{0}(u) \cap B_{1 / 2}\right) \leq C
$$

where the positive constant $C$ depends only on the manifold $N$ and the constant $E$. This is the case if $\operatorname{sing}(u)=\phi$. Now if $|D u|_{L^{\infty}\left(B_{1}\right)} \leq 1$, then $u$ is a smooth harmonic map. We apply Theorem A to $u$ to get a similar estimate with the new constant $C$ depending on the manifold and the quantity $\int_{B_{1}}\left|u-u_{1}\right|^{2}$.

4. Purterbations of Zeroes. The following result is used in the proof of Lemma 2.5 and 3.3. It was proved in [17]. We just point out some key steps.

LEMma 4.1. Let $P$ be a homogeneous harmonic polynomial of degree $d \geq 2$ and of two variables in $\mathbb{R}^{n}$. Then there exist positive constants $\varepsilon$ and $r$, depending on $P$, such that for any $u \in C^{2 d^{2}}\left(B_{1}\right)$ if

$$
|u-P|_{C^{2 d^{2}}\left(B_{1}\right)}<\varepsilon
$$

then

$$
\mathcal{H}^{n-2}\left(|D u|^{-1}\{0\} \cap B_{r}\right) \leq c(n)(d-1)^{2} r^{n-2}
$$

The proof is based on the Weierstrass-Malgrange Preparation Theorem for finitely differentiable functions. First we recall some terminology. For any point $p \in \mathbb{R}^{n}$ we let $C_{p}^{\infty}\left(\mathbb{R}^{n}\right)$ denote the ring of germs of smooth functions in a neighborhood of $p$. For a smooth map $f$ from a neighborhood of $p$ into $\mathbb{R}^{n}$ with $f(p)=0$ we let $(f)$ denote the ideal generated by $f_{1}, \cdots, f_{n}$, the components of $f$. The local ring of $f$ at $p$ is the quotient ring

$$
\mathcal{R}_{f}(p)=C_{p}^{\infty}\left(\mathbb{R}^{n}\right) /(f) .
$$

It is easy to see that $\mathcal{R}_{f}(p)$ is a vector space over $\mathbb{R}$, whose dimension is called the multiplicity of $f$ at $p$. Instead of $C_{p}^{\infty}\left(\mathbb{R}^{n}\right)$ in the above definition we may also use $\mathbb{P}\left(\mathbb{R}^{n}\right)$, the space of all polynomials in $\mathbb{R}^{n}$, or $C_{p}^{\omega}\left(\mathbb{R}^{n}\right)$, the space of analytic germs at $p$. See [1] or [13]. The above notion can be defined for functions in $\mathbb{C}^{n}$. The importance of multiplicity is its connection with zeroes of maps. It can be shown that holomorphic maps, which maps zero to zero, have finite multiplicity at the origin if and only if the origin is the isolated zero point. This result is not true in $\mathbb{R}^{n}$, even for analytic maps.

The notion of local rings and multiplicities can also be defined for finitely differentiable functions.

The following result was proved in [3]. We assume that $\mu, N$ and $N^{\prime}$ are all positive integers with $N \leq N^{\prime}$.

Lemma 4.2. Let $D \subset \mathbb{R}^{n}$ be a domain with $0 \in D$. Let $f: D \rightarrow \mathbb{R}^{n}$, with $f(0)=0$, be a function of smoothness $\mu\left(N^{\prime}+1\right)$ with the multiplicity $\mu$ at 0 , and let $\left\{e_{1}, \cdots, e_{\mu}\right\}$ be a basis of its local ring consisting of functions of smoothness $\mu(N+1)$. Then there exist neighborhoods $U, V$ and $Q$ of zero in $\mathbb{R}^{n}$, for which $V \subset U \subset D$ and 
$f(V) \subset Q \subset f(U)$, and a positive constant $\varepsilon$ with the following property: for any map $g: D \rightarrow \mathbb{R}^{n}$ of smoothness $\mu\left(N^{\prime}+1\right)$, if

$$
|f-g|_{C^{\mu\left(N^{\prime}+1\right)}(D)}<\varepsilon
$$

there exists a bounded linear operator

$$
E^{g}=\left(E_{1}^{g}, \cdots, E_{\mu}^{g}\right): C^{\mu(N+1)}(U) \rightarrow\left[C^{N}(Q)\right]^{\mu},
$$

such that for any function $\varphi \in C^{N}(U)$ there holds

$$
\left.\varphi\right|_{V}=\sum_{i=1}^{\mu} e_{i} \cdot\left(E_{i}^{g} \varphi\right) \circ g .
$$

Proof of Lemma 4.1. We first prove for $n=2$. By using the polar coordinate $x_{1}=r \cos \theta$ and $x_{2}=r \sin \theta$ in $\mathbb{R}^{2}=\left\{\left(x_{1}, x_{2}\right)\right\}$ we may assume $P(x)=r^{d} \cos d \theta$. Direct calculation shows that

$$
D_{x_{1}} P=d r^{d-1} \cos (d-1) \theta, D_{x_{2}} P=-d r^{d-1} \sin (d-1) \theta .
$$

Therefore both $D_{x_{1}} P$ and $D_{x_{2}} P$ are products of $d-1$ different homogeneous linear functions. We obtain that the map $f=\left(D_{x_{1}} P, D_{x_{2}} P\right): \mathbb{R}^{2} \rightarrow \mathbb{R}^{2}$ has the origin as its only zero. In fact if $f$ is viewed as a map from $\mathbb{C}^{2}$ to $\mathbb{C}^{2}$, with $x \in \mathbb{R}^{2}$ replaced by $z \in \mathbb{C}^{2}$, the origin is also its only zero. Hence by Bezout's formula ([1], Corollary 1 , P200) we conclude that

$$
\operatorname{dim} \mathbb{P}\left(\mathbb{R}^{2}\right) /(f) \leq(d-1)^{2},
$$

where $\mathbb{P}\left(\mathbb{R}^{2}\right)$ is the space of all polynomials in $\mathbb{R}^{2}$.

We may apply Lemma 4.2 with $N^{\prime}=N=1$ and $\mu=(d-1)^{2}$. We obtain that there exist neighborhoods $U, V, Q$ of the origin in $\mathbb{R}^{2}$ with $V \subset U \subset B_{1}$ and $f(V) \subset Q$ and a positive constant $\varepsilon>0$ such that for any map $g \in C^{2(d-1)^{2}}\left(B_{1} ; \mathbb{R}^{2}\right)$ with $|g-f|_{C^{2(d-1)^{2}\left(B_{1}\right)}}<\varepsilon$ and any function $a \in C^{2(d-1)^{2}}(U)$ there exist $\alpha_{1}, \cdots, \alpha_{\mu} \in$ $C^{1}(Q)$ such that

$$
a(x)=\sum_{i=1}^{\mu} e_{i}(x) \alpha_{i}(g(x)) \quad \text { for } x \in V .
$$

Hence for such a map $g$ we may prove for some positive constant $r$ with $B_{r} \subset V$

$$
\operatorname{Card}\left(g^{-1}\{0\} \cap B_{r}\right) \leq(d-1)^{2} .
$$

The proof is a modification of that for Lemma 2, P97, in [1].

Now consider $u \in C^{2 d^{2}}\left(B_{1}\right)$ with $|u-P|_{C^{2 d^{2}\left(B_{1}\right)}}<\varepsilon$. Note $2(d-1)^{2}+1 \leq 2 d^{2}$ for any positive integer $d$. Hence with $g=D u$ we have $|g-f|_{C^{2(d-1)^{2}\left(B_{1}\right)}}<\varepsilon$. Therefore we conclude

$$
\operatorname{Card}\left(|D u|^{-1}\{0\} \cap B_{r}\right) \leq(d-1)^{2} .
$$

This finishes the proof for $n=2$.

Next we discuss the general dimension. For any $p \in \mathbb{R}^{n}$ and any $1 \leq i<j \leq n$ let $\mathbb{P}_{i j}(p)$ denote the 2-dimensional hyperplane

$$
\left\{\left(p_{1}, \cdots, p_{i-1}, x_{i}, p_{i+1}, \cdots, p_{j-1}, x_{j}, p_{j+1}, \cdots, p_{n}\right)\right\}
$$


and simply write $\mathbb{P}_{i j}(p)=\left\{\left(x_{i}, x_{j}\right)\right\}$ where there is no confusion. We also set $\mathbb{P}_{i j}=$ $\mathbb{P}_{i j}(0)$.

Now let $P$ be a homogeneous harmonic polynomial of degree $d$ and of two variables in $\mathbb{R}^{n}$. With the explicit expression of $P$ we may find a change of coordinates with the following property. In the new coordinate system $\left\{\left(x_{1}, \cdots, x_{n}\right)\right\}$, for any fixed $1 \leq i<j \leq n$, the map $f_{i j}=\left(D_{x_{i}} P, D_{x_{j}} P\right) \mid \mathbb{P}_{i j}$, viewed as a map from $\mathbb{R}^{2}$ to $\mathbb{R}^{2}$, has the origin as its only zero and each component of $f_{i j}$ is the product of $d-1$ homogeneous linear polynomials. In fact if $f_{i j}$ is viewed as a map from $\mathbb{C}^{2}$ to $\mathbb{C}^{2}$, with $x \in \mathbb{R}^{n}$ replaced by $z \in \mathbb{C}^{n}$, the origin is also its only zero. As before there exist positive constants $\varepsilon_{i j}$ and $r_{i j}$ such that for any $g \in C^{2(d-1)^{2}}\left(B_{1 / 2}^{2} ; \mathbb{R}^{2}\right)$ with

$$
\left|g-f_{i j}\right|_{C^{2(d-1)^{2}}\left(B_{1 / 2}^{2}\right)}<\varepsilon_{i j}
$$

there holds

$$
\operatorname{card}\left(g^{-1}\{0\} \cap B_{r_{i j}}^{2}\right) \leq(d-1)^{2} .
$$

Here we use $B_{r}^{2}$ to denote the ball (centered at origin) with radius $r$ in $\mathbb{R}^{2}$.

Take

$$
\varepsilon=\frac{1}{2} \min _{1 \leq i<j \leq n} \varepsilon_{i j}, \quad r=\min _{1 \leq i<j \leq n} r_{i j} .
$$

Consider any $u \in C^{2 d^{2}}\left(B_{1}\right)$ such that

$$
|u-P|_{C^{2 d^{2}\left(B_{1}\right)}}<\varepsilon .
$$

For any $p \in \mathbb{R}^{n}$ and any $1 \leq i<j \leq n$, set $f_{i j}=\left(D_{x_{i}} P, D_{x_{j}} P\right) \mid \mathbb{P}_{i j}$ as before and $g_{i j, p}=\left(D_{x_{i}} u, D_{x_{j}} u\right) \mid \mathbb{P}_{i j}(p)$. We may take $r$ smaller such that for any $p \in B_{r}$ there holds

$$
\left|g_{i j, p}-f_{i j}\right|_{C^{2(d-1)^{2}}\left(B_{1 / 2}^{2}\right)}<2 \varepsilon \leq \varepsilon_{i j}
$$

Hence

$$
\operatorname{card}\left(g_{i j, p}^{-1}\{0\} \cap B_{r}^{2}\right) \leq(d-1)^{2} .
$$

Obviously $|D u|^{-1}\{0\} \cap \mathbb{P}_{i j}(p) \subset g_{i j, p}^{-1}\{0\}$. If we set $\pi_{i j}$ as the projection

$$
\pi_{i j}\left(x_{1}, \cdots, x_{n}\right)=\left(x_{1}, \cdots, x_{i-1}, x_{i+1}, \cdots, x_{j-1}, x_{j+1}, \cdots, x_{n}\right) \in \mathbb{R}^{n-2}
$$

then we have shown that for any $q \in B_{r}^{n-2} \subset \mathbb{R}^{n-2}$ and any $1 \leq i<j \leq n$

$$
\operatorname{card}\left(|D u|^{-1}\{0\} \cap \pi_{i j}^{-1}(q) \cap B_{r}\right) \leq(d-1)^{2} .
$$

Hence the integral geometric formula [10], 3.2.22, implies

$$
\begin{aligned}
& \mathcal{H}^{n-2}\left(|D u|^{-1}\{0\} \cap B_{r}\right) \\
\leq & \sum_{1 \leq i<j \leq n} \int_{B_{r}^{n-2}} \operatorname{card}\left(|D u|^{-1}\{0\} \cap \pi_{i j}^{-1}(q) \cap B_{r}\right) d \mathcal{H}^{n-2} q \\
\leq & c(n)(d-2)^{2} r^{n-2} .
\end{aligned}
$$


5. Geometric Structure and Measure of Rank One Sets. In the final section, we discuss the rank one sets for harmonic maps, both smooth and minimizing. Since the discussion follows the same line for the rank zero sets, we only point out the difference.

As in Section 1 , we assume that $B_{1}$ is the unit ball in $\mathbb{R}^{n}, n \geq 2$, endowed with the standard Euclidean metric. We further assume that $(N, g)$ is a smooth compact Riemannian manifold of the dimension $m \geq 2$. Throughout this section we will not view the manifold $N$ isometrically embedded in some Euclidean space. For a smooth map $u: B_{1} \rightarrow N$, with respect to local coordinates $x \mapsto u$, the gradient $D u(p)$ can be represented by the matrix $u_{i}^{\alpha}=\frac{\partial u^{\alpha}}{\partial x^{i}}$.

Suppose that $u: B_{1} \rightarrow N$ is a smooth harmonic map. Then $u$ satisfies the system

$$
\triangle u^{\alpha}+\sum_{i=1}^{n} \Gamma_{\beta \gamma}^{\alpha} u_{i}^{\beta} u_{i}^{\gamma}=0
$$

where $\Gamma$ 's are the Christoffel symbols on $(N, g)$. We define the rank one set $\mathcal{R}_{1}(u)$ as

$$
\mathcal{R}_{1}(u)=\left\{p \in B_{1} ; D u(p) \text { has rank one }\right\} .
$$

For a minimizing harmonic map $u: B_{1} \rightarrow N$, we define the rank one set $\mathcal{R}_{1}(u)$ as

$$
\mathcal{R}_{1}(u)=\{p \in \operatorname{reg}(u) ; D u(p) \text { has rank one }\},
$$

where $\operatorname{reg}(u)$ denotes the regular set of $u$, the subset of $B_{1}$ where $u$ is smooth. As pointed out in Section 1, it is known that

$$
\operatorname{dim}_{\mathcal{H}}\left(B_{1} \backslash \operatorname{reg}(u)\right) \leq n-3 .
$$

We first discuss the geometric structure of rank one sets.

Theorem 5.1. Suppose $u \in H^{1}\left(B_{1}, N\right)$ is a smooth harmonic map or a minimizing harmonic map. Then the rank one set $\mathcal{R}_{1}(u)$ is countably $(n-1)$-rectifiable unless $u$ maps the whole $B_{1}$ to either a point or a geodesic in $N$.

Proof. Since this is a local result, we may assume that $u$ is smooth in $B_{1}$. Hence $u$ satisfies the elliptic system (5.1). By the unique continuation property of the secondorder elliptic systems, (see [2]), $u$ cannot vanish to infinite order unless $u$ is a constant. In the following we always assume that $u$ is not a constant map or a geodesic map, i.e., $u\left(B_{1}\right)$ is not a geodesic in $N$.

Take $0 \in \mathcal{R}_{1}(u)$. As in the proof of Theorem 1.1, we will use the leading polynomials of $u$ at 0 to study the rank one set $\mathcal{R}_{1}(u)$ near 0 . We claim that by choosing appropriate coordinate system $\left\{x^{i}\right\}$ in $\mathbb{R}^{n}$ and $\left\{y^{\alpha}\right\}$ at $u(0)$ in $N$, the harmonic map $u$ has the following Taylor expansion of $u$ at 0

$$
u^{\alpha}=P_{\alpha}+R_{\alpha} \text { for } \alpha=1, \cdots, m,
$$

where $P_{1}(x)=c x_{1}$ for some constant $c \neq 0$ and $P_{2}, \cdots, P_{m}$ are homogeneous harmonic polynomials with degree $d \geq 2$, at least one of which is not identically zero.

To prove the claim, we note that $D u(0): R^{n} \rightarrow T_{u(0)} N$ has rank one as a linear transform. So we can choose a coordinate system $y^{\alpha}$ at $u(0)$ such that

$$
g_{u(0)}\left(D u, d y^{\alpha}\right)=0 \text { for any } \alpha \neq 1
$$


Let $\sigma$ be the geodesic in $N$ through $u(0)$ in the direction $\frac{\partial}{\partial y_{1}}$. Then one conclude that $\Gamma_{11}^{\alpha}(y) \equiv 0$ for any $y \in \sigma$ near $u(0)$. Furthermore, we can choose the usual Fermi coordinate system along $\sigma$; in other words, we require that $\Gamma_{\beta \gamma}^{\alpha}(y)=0$ for any $\alpha>1$ and any $y \in \sigma^{-}$near $u(0)$. Thus there holds for any $\alpha \geq 2$

$$
\left|\Gamma_{\beta \gamma}^{\alpha}(y)\right|=O\left(\left(0, y_{2}, \cdots, y^{m}\right)\right) \leq \sum_{\alpha \geq 2}\left|y^{\alpha}\right|
$$

By the system (5.1), we have for any $\alpha \geq 2$

$$
\begin{aligned}
\left|\triangle u^{\alpha}\right| & =\left|\sum_{i=1}^{n} \Gamma_{\beta \gamma}^{\alpha}(u) u_{i}^{\beta} u_{i}^{\gamma}\right| \\
& =O\left(\left|\left(0, u^{2}, \cdots, u^{m}\right)\right|\right)|D u|^{2} \leq C \sum_{\beta \geq 2}\left|u^{\beta}\right|,
\end{aligned}
$$

where $C$ is a constant depending on the derivatives of $u$ as well as the geometry of $N$. By the unique continuation property, (see [2]), either $\left(u^{2}, \cdots, u^{m}\right)(x) \equiv 0$ for $x$ near 0 or $\left(u^{2}, \cdots, u^{m}\right)$ vanishes at 0 up to finite order. However $\left(u^{2}, \cdots, u^{m}\right)(x) \equiv 0$ implies that the image of the original map $u(x)=\left(u^{1}, u^{2}, \cdots, u^{m}\right)(x)$ near 0 is contained in the set $\left\{y^{1}=0\right\}$, i.e., the geodesic $\sigma$. By the unique continuation property again, the whole image of the map $u$ is contained in $\sigma$. This is a contradiction to the hypothesis. Therefore $\left(u^{2}, \cdots, u^{m}\right)$ has a nontrivial Taylor expansion at 0 . We assume the leading terms are given by homogeneous polynomial $\left(P_{2}, \cdots, P_{m}\right)$ of degree $d \geq 2$. At least one of the $P_{\alpha}$ is not identically zero. Proceed as in the proof of Theorem 1.1, all $P_{\alpha}$ are harmonic polynomials, $\alpha=2, \cdots, m$. Note that $u^{1}$ has a nontrivial linear term by the construction. This finishes the proof of the claim.

As in the proof of Theorem 1.1, in order to study the rank one set $\mathcal{R}_{1}(u)$ of $u$ near 0 , we just need to study the rank one set $\mathcal{R}_{1}(P)$ of $P=\left(P_{1}, P_{2}, \cdots, P_{m}\right)$, where $P_{1}(x)=x_{1}$ and $P_{2}, \cdots, P_{m}$ are homogeneous harmonic polynomials with degree $d \geq 2$, at least one of which is not identically zero. Since $D P_{1}(x)=(1,0, \cdots, 0)$, it is easy to see that

$$
\mathcal{R}_{1}(P)=\left\{x \in R^{n} ; \frac{\partial P_{\alpha}}{\partial x_{i}}=0, \text { for any } \alpha=2, \cdots, m, \text { and } i=2, \cdots, n\right\} .
$$

Suppose for some $2 \leq \alpha \leq m$,

$$
\frac{\partial P_{\alpha}}{\partial x_{i}} \equiv 0, \text { for any } i=2, \cdots, n \text {. }
$$

Then $P_{\alpha}$ is a harmonic function of variable $x_{1}$, hence $P_{\alpha}$ must constant or linear. This contradicts the fact $\operatorname{deg}\left(P_{\alpha}\right)=d \geq 2$ if $P_{\alpha}$ is not identically zero. Therefore if $P_{\alpha}$ is not identically zero, then for some $2 \leq i \leq n, \frac{\partial P_{\alpha}}{\partial x_{i}}$ is not an identically zero function. For such $\alpha$ and $i$,

$$
\mathcal{R}_{1}(P) \subset\left\{x \in \mathbb{R}^{n} ; \frac{\partial P_{\alpha}}{\partial x_{i}}(x)=0\right\}
$$

Note $\left\{x \in \mathbb{R}^{n} ; \frac{\partial P_{\alpha}}{\partial x_{i}}(x)=0\right\}$ is a real analytic variety of dimension $\leq n-1$. Now proceed as in the proof of Theorem 1.1, we may obtain a similar stratification result for $\mathcal{R}_{1}(u)$. In particular, $\mathcal{R}_{1}(u)$ is countably $(n-1)$-rectifiable near 0 . This finishes the proof. 
Next we discuss how to obtain the measure estimates of rank one sets of harmonic maps. As suggested by Theorem 1.1, we need to describe how harmonic maps differ from geodesic maps quantitatively. One way is to consider all the possible difference with geodesic map as follows:

$$
c(u)=\inf \left\{|u-\sigma|_{L^{\infty}\left(B_{1}\right)} ; \sigma: B_{1} \rightarrow N \text { is a geodesic map }\right\} .
$$

Obviously $u$ is not a geodesic map if and only if $c(u)>0$. An alternative way is to introduce the following section $J(u)$ on the bundle $\Gamma^{\infty}\left(\left[u^{*}\left(\wedge^{2} T^{*} N\right) \otimes \wedge^{2} \mathbb{R}^{n}\right]^{*}\right)$ :

$$
J(u)_{p}\left(w_{1} \wedge w_{2}\right)\left(v_{1} \wedge v_{2}\right)=\left[u^{*}\left(w_{1} \wedge w_{2}\right)\right]\left(v_{1} \wedge v_{2}\right)
$$

where $w_{1}, w_{2}$ are one-form in $T_{u(p)}^{*} N$, and $v_{1}, v_{2}$ are vectors on $\mathbb{R}^{n}$. It is easy to check that the union $\mathcal{R}_{0}(u) \cup \mathcal{R}_{1}(u)$ are exactly the collection of points $p$ such that $J(u)_{p}=0$. In fact $J(u)_{p}=0$ implies that for any $w_{1}, w_{2} \in T_{u(p)}^{*} N$ vectors $u^{*} w_{1}, u^{*} w_{2}$ are linearly independent in $T_{p} \mathbb{R}^{n} \cong \mathbb{R}^{n}$, which is equivalent to say that any $2 \times 2$ submatrix of the differential $D u$ at $p$ with respect to some coordinate systems for $\mathbb{R}^{n}$ and $T_{u(p)} N$ is always degenerate. Thus it describes a point $p$ in $\mathcal{R}_{0}(u) \cup \mathcal{R}_{1}(u)$.

The key step for the measure estimates of rank one sets is the following result. Compare with Lemma 2.5.

LEMMA 5.2. Suppose $u: B_{1} \rightarrow N_{1}$ is a smooth harmonic map, but not a geodesic map. Then there exists a finite collection of balls $\left\{B_{r_{i}}\left(x_{i}\right)\right\}$ with $x_{i} \in \mathcal{R}_{0}(u)$ and $r_{i} \leq 1 / 4$ such that

$$
H^{n-1}\left(\mathcal{R}_{1}(u) \cap B_{1 / 2} \backslash \cup B_{r_{i}}\left(x_{i}\right)\right) \leq C
$$

and

$$
\sum r_{i}^{n-1} \leq 1 / 2
$$

where $C$ is a positive constant depending only on the manifold $N$, the supnorm of $D u$ in $B_{1}$ and the reciprocal of $c(u)$ or the reciprocal of supnorm of $J(u)$ in $B_{1}$.

Proof. We only give a sketch of the proof since it proceeds similar to that of Lemma 2.5. Note $\mathcal{R}_{1}(u)$ is not a closed subset of $B_{1}$. The union $\mathcal{R}_{0}(u) \cup \mathcal{R}_{1}(u)$ is closed. By Theorem 1.1 we have

$$
H^{n-1}\left(\mathcal{R}_{0}(u)\right)=0 .
$$

Then there exist at most countably many balls $B_{r_{i}}\left(x_{i}\right)$ with $r_{i} \leq 1 / 8$ and $x_{i} \in \mathcal{R}_{0}(u)$ such that

$$
\mathcal{R}_{0}(u) \subset \bigcup_{i} B_{r_{i}}\left(x_{i}\right)
$$

and

$$
\sum r_{i}^{n-1} \leq \frac{1}{2^{n}}
$$

We claim for any $p \in \mathcal{R}_{1}(u) \cap B_{3 / 4}$, there exist positive constants $r=r(p, u)<$ $1 / 8, \eta=\eta(p, u), c=c(p, u)$ and positive integer $d=d(p, u)$ such that if the smooth map $v: B_{1} \rightarrow N$ satisfies

$$
|u-v|_{C^{d}\left(B_{r}(p)\right)}<\eta
$$


then

$$
H^{n-1}\left\{\mathcal{R}_{1}(v) \cap B_{r}(p)\right\} \leq c r^{n-1} .
$$

To prove this we employ the Taylor expansion of $u$ we derived in the proof of Theorem 5.1. For $p \in \mathcal{R}_{1}(u) \cap B_{3 / 4}$, by taking appropriate coordinate system $\left\{x^{i}\right\}$ in $\mathbb{R}^{n}$ and $\left\{y^{\alpha}\right\}$ at $u(p)$ in $N$, the harmonic map $u$ has the following Taylor expansion of $u$ at 0

$$
u^{\alpha}(\cdot+p)=P_{\alpha}+R_{\alpha} \text { for } \alpha=1, \cdots, m,
$$

where $P_{1}(x)=\gamma x_{1}$ for some constant $\gamma \neq 0$ and $P_{2}, \cdots, P_{m}$ are homogeneous harmonic polynomials with degree $d \geq 2$, at least one of which is not identically zero. We assume $P_{2}$ is not identically zero. Moreover by the argument in the proof of Theorem $5.1, \frac{\partial P_{2}}{\partial x_{i}}$ is not an identically zero function for some $i=2, \cdots, m$, say $i=2$. Since $\frac{\partial P_{2}}{\partial x_{2}}$ is a nonzero homogeneous polynomial of degree $d-1$, we may find an orthonormal basis $\left\{e_{1}, \cdots, e_{n}\right\}$ in $\mathbb{R}^{n}$ such that

$$
D_{e_{i}}^{d-1}\left(\frac{\partial P_{2}}{\partial x_{2}}\right) \text { is a nonzero constant for any } i=1, \cdots, n \text {. }
$$

For any smooth map $v: B_{1} \rightarrow N$ close to $u$ near $p$, the rank one set $\mathcal{R}_{1}(v)$ is contained in the zero set of the function

$$
\tilde{v} \equiv \operatorname{det}\left(\frac{\partial v^{\alpha}}{\partial x^{i}}\right)_{\{\alpha, i=1,2\}}=\frac{\partial v^{1}}{\partial x^{1}} \cdot \frac{\partial v^{2}}{\partial x^{2}}-\frac{\partial v^{1}}{\partial x^{2}} \cdot \frac{\partial v^{2}}{\partial x^{1}} .
$$

The corresponding function for $P$ is

$$
\tilde{P} \equiv \operatorname{det}\left(\frac{\partial P^{\alpha}}{\partial x^{i}}\right)_{\{\alpha, i=1,2\}}=\frac{\partial P^{1}}{\partial x^{1}} \cdot \frac{\partial P^{2}}{\partial x^{2}}-\frac{\partial P^{1}}{\partial x^{2}} \cdot \frac{\partial P^{2}}{\partial x^{1}}=\gamma \frac{\partial P_{2}}{\partial x_{2}} .
$$

Therefore there exist positive constants $r=r(p, P)<1 / 8$ and $\eta=\eta(p, P)$ such that if the smooth map $v: B_{1} \rightarrow N$ satisfies

$$
|P-v(p+\cdot)|_{C^{d}\left(B_{r}(0)\right)}<\eta,
$$

then

$$
D_{e_{i}}^{d-1} \tilde{v} \text { is never zero in } B_{r}(p) \text { for any } i=1, \cdots, n \text {. }
$$

By using 1-dimensional mean value theorem $d-1$ times, we conclude that there cannot be more that $d$ zeroes of $\tilde{v}$ in any line parallel to $e_{i}$ for some $i$. Hence integral geometric formula implies

$$
H^{n-1}\left\{\tilde{v}^{-1}(0) \cap B_{r}(p)\right\} \leq c r^{n-1},
$$

where $c$ is constant depending on $p$ and $P$. By the inclusion $\mathcal{R}_{1}(v) \subset \tilde{v}^{-1}(0)$, this proves (5.8) under the assumption (5.7).

The rest of the proof proceeds exactly as in Lemma 2.5, with (2.3)-(2.6) replaced by (5.5)-(5.8).

By Lemma 5.2 we may derive Theorem $\mathrm{C}$ in the same way we prove Theorem A. We may also prove a similar result as Lemma 5.2 for minimizing harmonic maps and then prove Theorem $\mathrm{D}$. We omit the details.

As a final remark, we point out that the discussion of rank one sets can be extended to stationary harmonic maps. For stationary harmonic maps the singular sets have $(n-2)$-dimensional Hausdorff measure. So they have the lower dimension 
compared with rank one sets. Hence the discussion is similar to that for the minimizing harmonic maps as long as the weak limits are not constant maps or geodesic maps.

\section{REFERENCES}

[1] V. I. Arnold, S. M. Gusein-Zade, and A. N. Varchenko, Singularities of Differentiable Maps, Volume I, Birkhauser, 1985.

[2] N. Aronszajn, A unique continuation theorem for solutions of elliptic differential equations or inequalities of second order, J. Math. Pures et Appl., T. 36 (1957), pp. 235-249.

[3] V. I. BAKHTin, Weierstrass-Malgrange preparation theorem for finitely smooth case, Functional Anal. Appl., 24 (1990), pp. 86-96.

[4] V. I. BAKHTIN, Weierstrass preparation theorem for finitely smooth modules, in Theory of Singularities and its Applications, Advances in Soviet Mathematics 1, pp. 287-294.

[5] L. Bers, Local behavior of solution of general linear elliptic equations, Comm. Pure Appl. Math., 8 (1955), pp. 473-496.

[6] L. A. CAffarelli AND A. Friedman, Partial regularity of the zero-set of solutions of linear and superlinear elliptic equations, J. Diff. Eq., 60 (1985), pp. 420-433.

[7] J. P. D'Angelo, Several Complex Variables and the Geometry of Real Hypersurfaces, CRC Press, Inc., 1992.

[8] H. Donnelly and C. Fefferman, Nodal sets of eigenfunctions on Riemannian manifolds, Invent. Math., 93 (1988), pp. 161-183.

[9] H. DONNElly AND C. FEFFERMAN, Nodal sets for eigenfunctions of the Laplacian on surfaces, J. Amer. math. Soc., 3 (1990), pp. 333-353.

[10] H. Federer, Geometric Measure Theory, Springer-Verlag, New York, 1969.

[11] N. Garofalo and F.-H. Lin, Monotonicity properties of variational integrals, $A_{p}$ weights and unique continuation, Indiana Univ. Math J., 35 (1986), pp. 245-267.

[12] D. Gilbarg and N. Trudinger, Elliptic Partial Differential Equations of Second Order, Second Edition, Springer, Berlin, 1983.

[13] M. Golubitsky and V. Gulllemin, Stable Mappings and Their Singularities, SpringerVerlag, New York, 1973.

[14] M. Gromov AND R. Schoen, Harmonic maps into singular spaces and p-adic superrigidity for latices in groups of rank one, IEHS Publications.

[15] Q. HAN, Singular sets of solutions to elliptic equations, Indiana Univ. Math J., 43 (1994), pp. 983-1002.

[16] Q. HAN, Pointwise Schauder estimates with applications to nodal sets, to appear.

[17] Q. HAN, R. HARDT, AND F.-H. LIN, Geometric measure of singular sets of elliptic equations, Comm. Pure Appl. Math., 51 (1998), pp. 1425-1443.

[18] Q. HAN AND F.-H. Lin, On the geometric measure of nodal sets of solutions, J Partial Diff. Eq., 7 (1994), pp. 111-131.

[19] R. HARDT AND L. Simon, Nodal sets for solutions of elliptic equations, J. Diff. Geom. 30 (1989), pp. 505-522.

[20] M. Hoffman-Ostenhof, T. Hoffman-Ostenhof, and N. Nadirashvili, Critical sets of smooth solutions to elliptic equations in dimension 3, Indiana Univ. Math J., 45 (1996), pp. 15-37.

[21] M. Hoffman-Ostenhof, T. Hoffman-Ostenhof, N. Nadirashvili, and R. Hardt, Critical sets of smooth solutions to elliptic equations, to appear.

[22] F.-H. LiN, Nodal sets of solutions of elliptic and parabolic equations, Comm. Pure Appl. Math., 45 (1991), pp. 287-308.

[23] F.-H. LIN, Gradient estimates and blow-up analysis for stationary harmonic maps, I, to appear.

[24] B. Malgrange, Ideals of Differentiable Mappings, Oxford Univ. Press, Oxford, 1966.

[25] R. Narasimhan, Introduction to the Theory of Analytic Spaces, Lecture Notes in Mathematics 25, Springer-Verlag, Berlin, New York, 1966.

[26] J. H. SAMPSON, Some properties and applications of harmonic mappings, in Ann. Ec. Norm. Sup. 11, 1978, pp. 211-228.

[27] R. Schoen and K. Uhlenbeck, A regularity theory for harmonic maps, J. Diff. Geom., 17 
(1982), pp. 307-335.

[28] L. Simon, Lectures on Geometric Measure Theory, Proc. C.M.A., Aust. Nat. Univ., 1983.

[29] S. YAMADA, On the ranks of harmonic maps, preprint.

[30] S. T. YAU, Open problems in geometry, Proc. Symp. Pure Math., 54 (1993), pp. 1-28.

[31] Y. Yomdin, The set of zeroes of an almost polynomial functions, Proc. Amer. Math. Soc., 90:4 (1984), pp. 538-542. 